\title{
SOLUS: a novel multimodal approach to ultrasound and diffuse optics imaging of breast cancer
}

Pifferi, Antonio, Dalla Mora, Alberto, Di Sieno, Laura, Maffeis, Giulia, Tosi, Alberto, et al.

Antonio Pifferi, Alberto Dalla Mora, Laura Di Sieno, Giulia Maffeis, Alberto Tosi, Enrico Conca, Vincenzo Sesta, Andrea Giudice, Alessandro Ruggeri, Simone Tisa, Alexander Flocke, Bogdan Rosinski, Jean-Marc Dinten, Mathieu Perriollat, Hélène Sportouche, Christophe Fraschini, Simon Arridge, Giuseppe Di Sciacca, Andrea Farina, Pietro Panizza, Elena Venturini, Peter Gordebeke, Pamela Zolda, Paola Taroni, "SOLUS: a novel multimodal approach to ultrasound and diffuse optics imaging of breast cancer," Proc. SPIE 11920, Diffuse Optical Spectroscopy and Imaging VIII, 1192003 (9 December 2021); doi: $10.1117 / 12.2614309$ 


\title{
SOLUS: A Novel Multimodal Approach to Diffuse Optics and Ultrasound Imaging of Breast Cancer
}

\author{
Antonio Pifferia, ${ }^{a,}$, Alberto Dalla Mora ${ }^{a}$, Laura Di Sieno ${ }^{a}$, Giulia Maffeis ${ }^{a}$, Alberto Tosib ${ }^{b}$ Enrico Conca ${ }^{b}$, Vincenzo Sesta ${ }^{b}$, \\ Andrea Giudice $^{c}$, Alessandro Ruggeric ${ }^{\circ}$, Simone Tisa ${ }^{c}$, Alexander Flocke ${ }^{\mathrm{d}}$, Bogdan Rosinski ${ }^{\mathrm{e}}$, Jean-Marc Dinten ${ }^{\mathrm{f}}$, Mathieu \\ Perriollat $^{\mathrm{f}}$, Hélène Sportouche ${ }^{\mathrm{g}}$, Christophe Fraschini ${ }^{\mathrm{g}}$, Simon Arridge ${ }^{\mathrm{h}}$, Giuseppe Di Sciacca ${ }^{\mathrm{h}}$, Andrea Farina ${ }^{\mathrm{i}}$, Pietro \\ Panizza $^{j}$, Elena Venturini ${ }^{j}$, Peter Gordebeke ${ }^{k}$, Pamela Zolda ${ }^{k}$, Paola Taroni*a,i \\ ${ }^{a}$ Politecnico di Milano - Dipartimento di Fisica, Piazza Leonardo da Vinci 32, 20133 Milano (Italy); \\ ${ }^{b}$ Politecnico di Milano - Dipartimento di Elettronica, Informazione e Bioingegneria, Piazza Leonardo da Vinci 32, 20133 Milano (Italy); ${ }^{c}$ Micro Photon Devices

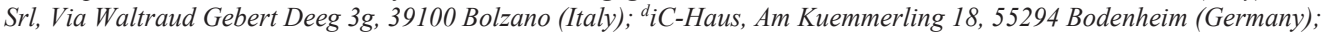 \\ ${ }^{e}$ Vermon S.A., 180 Rue du Général Renault, 37000, Tours (France); ${ }^{f}$ CEA-LETI, 17 Avenue des Martyrs, 38054 Grenoble, France; ${ }^{g}$ Supersonic Imagine S.A., 510 \\ rue René Descartes, Les jardins de la Duranne Bât. F, 13857 Aix en Provence (France); ${ }^{h}$ University College London, Department of Computer Science, Gower \\ Street WC1E 6BT London (UK); ${ }^{i}$ Consiglio Nazionale delle Ricerche, Istituto di Fotonica e Nanotecnologie, Piazza Leonardo da Vinci 32,20133 Milano (Italy); \\ ${ }^{j}$ Scientific Institute (IRCCS) Ospedale San Raffaele-Breast Imaging Unit, Via Olgettina 60, 20132 Milano (Italy); ${ }^{2}$ European Institute for Biomedical Imaging \\ Research, Am Gestade 1, 1010 Vienna (Austria) \\ paola.taroni@polimi.it
}

\begin{abstract}
A multimodal instrument for breast imaging was developed, combining ultrasound (morphology), shear wave elastography (stiffness), and time domain multiwavelength diffuse optical tomography (blood, water, lipid, collagen) to improve the non-invasive diagnosis of breast cancer. (C) 2021 The Author(s)
\end{abstract}

\section{Introduction}

Breast cancer is the most common female cancer, representing 1 in 4 cancers diagnosed among women globally [1]. Screening mammography is key for early diagnosis and effective treatment, but has weaknesses. An important one is its limited specificity, leading to a huge number of needless additional examinations, including invasive ones, like - typically - breast biopsy.

To improve the diagnostic accuracy, the combination of different x-ray-based techniques (e.g., conventional mammography and tomosynthesis [2]) as well as different imaging modalities (e.g., magnetic resonance imaging) are investigated .

\section{The SOLUS approach to breast imaging}

Each technique is sensitive to a specific characteristic of tissues and of their pathologic modifications, and that may limit its capability to discriminate healthy or benign tissues from malignant ones. The SOLUS project [3] proposes to achieve a more thorough and potentially effective characterization of tissue for diagnostic purposes combining techniques that investigate different tissue features: B-mode ultrasound (US) scanning (investigating morphology), Color Doppler imaging (CD, sensitive to vascularization), Shear Wave Elastography (SWE, to quantify stiffness), and time domain multi-wavelength Diffuse Optical Tomography (DOT, to assess tissue composition).

All techniques are performed through a single multimodal hand-held probe. US-based modalities are carried out through a regular US transducer, while the optical part of the system was fully designed and realized specifically for integration in the multimodal SOLUS probe.

\subsection{The smart optode}

The smart optode is the basic optical element of the multimodal probe, but is also an innovative, very compact (few $\left.\mathrm{cm}^{3}\right) \mathrm{stand}$ alone photonic device to perform a time domain multi-wavelength diffuse optical measurement that can be of interest for applications independent of medical imaging and diagnosis.

The optode contains:

- 8 picosecond pulsed laser diodes emitting at wavelengths selected in the range of 635-1064 $\mathrm{nm}$,

- A wide area fast-gated Silicon PhotoMultiplier (SiPM) detector,

- An integrated Time-to-Digital-Converter (TDC).

In the SOLUS probe, 8 optodes are arranged around the US transducers, as schematically shown in Fig. 2, to allow collection of light along different light paths for DOT reconstruction of the same volume that is imaged by US.

The optode components and the integrated device were fully characterized using recognized protocols for performance assessment (BIP, NEUROPT and MEDPHOT [4-6]). 


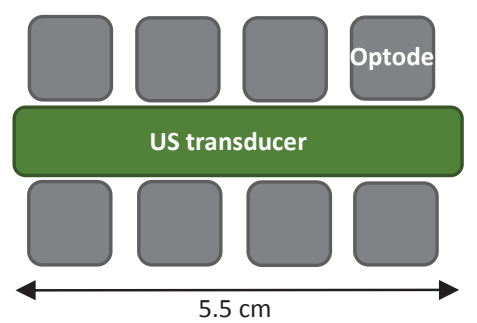

Fig. 1. Arrangement of the 8 optodes and the US transducer in the multimodal probe.

The laser drivers generate pulses with duration of the order of hundred of picoseconds (FWHM) up to a repetition rate of 80 $\mathrm{MHz}$, and both the pulse-width and delay can be tuned in 1 ps steps. Eight wavelengths were selected in the range of 635 to 1064 $\mathrm{nm}$, to allow effective probing of tissue composition in terms of blood parameters (total hemoglobin content and oxygenation level), water, lipid and collagen content. For pulse widths $<240 \mathrm{ps}$ (FWHM), average powers of 1.5 to $6 \mathrm{~mW}$ are obtained, depending on wavelength. The laser pulses show negligible secondary peaks or exponential tail.

Controllable active area and fast gating are key features of the digital SiPM detector [7] to allow operation with sourcedetector distances varying between $<1 \mathrm{~cm}$ and $>5 \mathrm{~cm}$, with collected signal ranging over several orders of magnitude. The active area can be increased from an individual single photon avalanche diode (SPAD, $<40 \mu \mathrm{m}^{2}$ ) up to a maximum of $8.6 \mathrm{~mm}^{2}$. The photon detection efficiency of each SPAD reaches $35 \%$ at $430 \mathrm{~nm}$ and decreases progressively for longer wavelengths. The gateopen transition is faster than $500 \mathrm{ps}$ up to an active area of $\approx 3 \mathrm{~mm}^{2}$. The temporal response also depends on the extension of the active area, ranging from $235 \mathrm{ps}$ (FWHM) for a single active cell to around $500 \mathrm{ps}$ at about $4 \mathrm{~mm}^{2}$.

The integrated TDC is paired with a 128-channel histogram builder and has average channel width (i.e., least significant bit) of $78 \mathrm{ps}$ and full-scale range of about $10 \mathrm{~ns}$. The dead time is $<100 \mathrm{~ns}$ and histograms can be transferred with no additional deadtime up to a rate of $30 \mathrm{kHz}$.

The full smart optode demonstrated very effective light harvesting, with responsivity $>10^{6} \mathrm{~m}^{2} \mathrm{sr}$ at $600 \mathrm{~nm}$ already for an active area of about $2 \mathrm{~mm}^{2}$.

It was tested for the assessment of optical properties of homogeneous media in the range of interest for in vivo measurements (absorption $\mu_{\mathrm{a}}=0.06-0.4 \mathrm{~cm}^{-1}$ and reduced scattering $\mu_{\mathrm{s}}{ }^{\prime}=4-17 \mathrm{~cm}^{-1}$ ). As an example, at $670 \mathrm{~nm}$ the absorption is retrieved with a relative error of $10 \%$ (median value), while the error on the scattering is much higher (up to $40 \%$ ). The reason for the latter unexpected negative behavior is under investigation.

The sensitivity to deep absorption inhomogeneities was also tested changing the depth of a perturbation $\left(\Delta \mu_{\mathrm{a}}=0.16 \mathrm{~cm}^{-1}\right.$ with respect to the surrounding medium). The perturbation could be detected down to a depth of $3.5 \mathrm{~cm}$ with $2 \%$ contrast, in line with what expected for real clinical measurements.

\subsection{The SOLUS system for multimodal imaging}

The full multimodal imaging system, shown in Fig. 2, was designed taking advantage of the Aixplorer ${ }^{\circledR}$ Mach 30, a high-end commercial instrument for US imaging from SuperSonic Imagine. The multimodal probe is connected to the Aixplorer and operation is controlled through a dedicated notebook and touchscreen.

The probe is water-cooled to provide constant working temperature and ensure controlled performance and safe temperature at the probe nose, in contact with the patient's skin. Class 1 lasers sources are exploited, making the imaging procedure fully noninvasive. This notwithstanding, dedicated sensors disable the lasers, when the probe is not in good contact with the skin.

Initial tests on a standard US phantom (ATS model 549) confirmed that the multimodal probe arrangement has no negative effect on the US imaging capability of the system as compared to what expected for a conventional US probe. The final calibration and tuning of the optical part are now being performed.

Data analysis for optical imaging foresees the following initial steps: i) manual segmentation performed on US images to identify the lesion; and ii) extraction of a 3D prior, which can be used to guide diffuse optical reconstructions, together with the enforcement of a spectral constrain, with the major aim of accurate estimate of tissue composition in the lesion as compared to the surrounding tissue. Several approaches to DOT in the SOLUS geometry were investigated and tested on simulations and phantom measurements. The adoption of a pure analytical model based on the Born approximation requires the availability of a reference measurement (e.g., obtained on the contra-lateral breast or on a region nearby the lesion). Alternatively, a FEM-based non-linear fitting would not require any reference measurement, but it would be more computationally intensive and time consuming. Both approaches will be challenged for the analysis of clinical data, considering the possibility to adopt the first one to provide a quick initial feedback to the operator, leaving further refinement (including non-linear fitting) to a subsequent offline stage. 


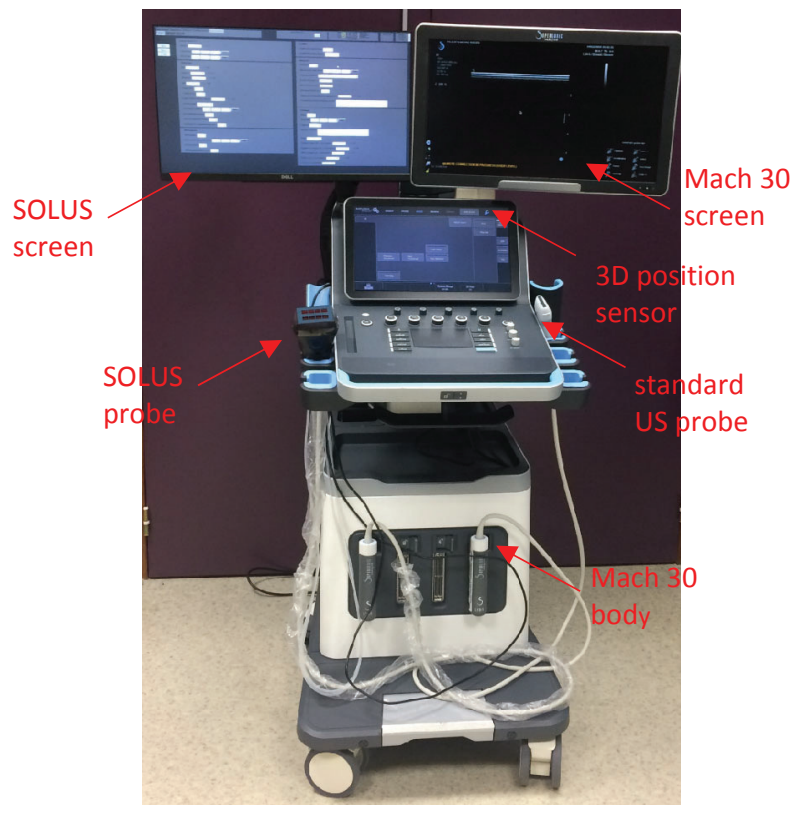

Fig. 2. SOLUS system for multimodal imaging.

\subsection{Conclusions}

An innovative system for non-invasive multimodal imaging of the breast was developed, combining B-mode US scans, DC imaging, SWE and multi-wavelength time domain DOT.

Full performance assessment will be carried out on phantoms, including dedicated bi-modal phantoms, heading to reach the phase of in vivo testing late in spring. Clinical validation will follow, including: i) mock sessions to train the medical doctors in the use of the multimodal probe and investigate usability and ergonomics; and ii) measurements on 40 patients with breast lesions (20 benign and 20 malignant ones) to start exploring the diagnostic capability of the SOLUS approach.

\section{References}

[1] IARC - International Agency for Research on Cancer., "GLOBOCAN 2020: New Global Cancer Data," < https://www.uicc.org/news/globocan-2020new-global-cancer-data> (14 February 2021 ).

[2] Giampietro, R. R., Cabral, M. V. G., Lima, S. A. M., Weber, S. A. T. and Dos Santos Nunes-Nogueira, V., "Accuracy and Effectiveness of Mammography versus Mammography and Tomosynthesis for Population-Based Breast Cancer Screening: A Systematic Review and Meta-Analysis," Sci. Rep. 10(1), 7991 (2020).

[3] "SOLUS - Smart Optical and Ultrasound Diagnostics of Breast Cancer.”, H2020 Proj. grant No. 731877, <http://solus-project.eu/>

[4] Wabnitz, H., Taubert, D. R., Mazurenka, M , Steinkellner, O., Jelzow, A., Macdonald, R., Milej, D., Sawosz, P., Kacprzak, M., Liebert, A., Cooper, R., Hebden, J., Pifferi, A., Farina, A., Bargigia, I., Contini, D., Caffini, M., Zucchelli, L., Spinelli, L., et al., "Performance assessment of time-domain optical brain imagers, part 1: basic instrumental performance protocol,” J. Biomed. Opt. 19(8), 86010 (2014).

[5] Wabnitz, H., Jelzow, A., Mazurenka, M., Steinkellner, O., Macdonald, R., Milej, D., Zolek, N., Kacprzak, M., Sawosz, P., Maniewski, R., Liebert, A., Magazov, S., Hebden, J., Martelli, F., Di Ninni, P., Zaccanti, G., Torricelli, A., Contini, D., Re, R., et al., "Performance assessment of time-domain optical brain imagers, part 2: nEUROPt protocol," J. Biomed. Opt. 19(8), 086012 (2014).

[6] Pifferi, A., Torricelli, A, Bassi, A., Taroni, P., Cubeddu, R., Wabnitz, H., Grosenick, D., Möller, M., Macdonald, R., Swartling, J., Svensson, T., Andersson-Engels, S., van Veen, R. L. P., Sterenborg, H. J. C. M., Tualle, J.-M., Nghiem, H. L., Avrillier, S., Whelan, M. and Stamm, H., "Performance assessment of photon migration instruments: the MEDPHOT protocol.," Appl. Opt. 44(11), 2104-2114 (2005).

[7] Conca, E., Sesta, V., Buttafava, M., Villa, F., Di Sieno, L., Dalla Mora, A., Contini, D., Taroni, P., Torricelli, A., Pifferi, A , Zappa, F. and Tosi, A., "Large-Area, Fast-Gated Digital SiPM with Integrated TDC for Portable and Wearable Time-Domain NIRS," IEEE J. Solid-State Circuits 55(11) (2020). 